\title{
Pyrosequencing Reveals Fungal Communities in the Rhizosphere of Xinjiang Jujube
}

\author{
Peng Liu, ${ }^{1}$ Xiao-Hui Wang, ${ }^{2}$ Jian-Gui Li, ${ }^{3}$ Wei Qin, ${ }^{3}$ Cheng-Ze Xiao, ${ }^{1}$ Xu Zhao, \\ Hong-Xia Jiang, ${ }^{2}$ Jun-Kang Sui, ${ }^{2}$ Rong-Bo Sa, ${ }^{2}$ Wei-Yan Wang, ${ }^{1}$ and Xun-Li Liu \\ ${ }^{1}$ College of Forest, Shandong Agricultural University, No. 61, Daizong Street, Taian, Shandong 271018, China \\ ${ }^{2}$ College of Life Science, Shandong Agricultural University, Taian, Shandong 271018, China \\ ${ }^{3}$ Research Institute of Forest in Xinjiang Agricultural University, Urumqi, Xinjiang 830052, China \\ Correspondence should be addressed to Xun-Li Liu; xlliu@sdau.edu.cn
}

Received 18 September 2014; Revised 26 November 2014; Accepted 29 November 2014

Academic Editor: Ernesto Picardi

Copyright (C) 2015 Peng Liu et al. This is an open access article distributed under the Creative Commons Attribution License, which permits unrestricted use, distribution, and reproduction in any medium, provided the original work is properly cited.

\begin{abstract}
Fungi are important soil components as both decomposers and plant symbionts and play a major role in ecological and biogeochemical processes. However, little is known about the richness and structure of fungal communities. DNA sequencing technologies allow for the direct estimation of microbial community diversity, avoiding culture-based biases. We therefore used 454 pyrosequencing to investigate the fungal communities in the rhizosphere of Xinjiang jujube. We obtained no less than 40,488 internal transcribed spacer (ITS) rDNA reads, the number of each sample was 6943, 6647, 6584, 6550, 6860, and 6904, and we used bioinformatics and multivariate statistics to analyze the results. The index of diversity showed greater richness in the rhizosphere fungal community of a 3-year-old jujube than in that of an 8-year-old jujube. Most operational taxonomic units belonged to Ascomycota, and taxonomic analyses identified Hypocreales as the dominant fungal order. Our results demonstrated that the fungal orders are present in different proportions in different sampling areas. Redundancy analysis (RDA) revealed a significant correlation between soil properties and the abundance of fungal phyla. Our results indicated lower fungal diversity in the rhizosphere of Xinjiang jujube than that reported in other studies, and we hope our findings provide a reference for future research.
\end{abstract}

\section{Introduction}

Fungi are important in soils as decomposers and plant symbionts [1]; it is thus important to understand the composition of their complex communities [2]. They play essential roles in many aspects of ecosystem development, function, and stability [3]. Understanding fungal diversity, community structure, and spatial patterns is one of the central issues in soil microbial ecology [4]. Microbial diversity is measured by a variety of techniques, including traditional plate culture, phospholipid and fatty acid analysis, phyloarrays, fingerprinting techniques, other DNA-based techniques such as restriction analysis and Sanger sequencing, and denaturing gradient gel electrophoresis of microbial proteins [5,6]. Plate culture is the primary method used to study microbial communities, but because of the methodological limitations associated with it $[7,8]$, only few studies have addressed the diversity of microbial communities. Although the vast majority of microbial soil species is unculturable, the development of culture-independent methods has yielded important insights into the phylogenetic diversity of soil microorganisms [9]. High-throughput sequencing technologies now play a significant role in microbial ecology studies [10] and nextgeneration techniques such as 454 pyrosequencing facilitate the detection of unculturable microbial communities [11, 12].

Pyrosequencing is faster, approximately 20-30 times less expensive than Sanger sequencing, and does not require cloning [13]. The 454 pyrosequencing technique has recently been used to characterize fungal diversity. Buée et al. [14] used this technique for forest soils to reveal unexpectedly high fungal diversity; Lim et al. [13] assessed soil fungal communities by using pyrosequencing, as did Jumpponen and Jones [15] to characterize the fungal communities in Quercus spp. Ectomycorrhizas and revealed seasonal dynamics in urban and rural sites. The 454 pyrosequencing platform is a new type of second-generation sequencing technology; 
it produces 25 million base reads in a single run with an accuracy of $99 \%$ [16]. It is rapid, flexible, and inexpensive, and it does not require a cloning step [17]. This technology permits metagenomic analyses that exceed the capacity of traditional Sanger sequencing-based approaches by several orders of magnitude and offers the possibility of detecting very rare phylotypes [18].

The Xinjiang Uygur Autonomous Region, which is the largest province in China, is situated in the hinterland of Asia and is characterized by very low precipitation and high evaporation [19]. These unique climatic characteristics of Xinjiang, such as little rainfall with periodic drought, abundant sunshine, and substantial differences between day and night temperatures [20], create conditions in which the composition and distribution of soil fungal communities may vary significantly. For example, Feng-gang et al. [21] analyzed the culturable fungal diversity in the rhizosphere of healthy and diseased cotton in southern Xinjiang. Chinese jujube is a common traditional Chinese medicine and food that has been used for thousands of years [22]. Jujube is one of the main economic crops in Xinjiang, as the quality of Xinjiang jujube is better than that in other regions because of the unique climatic conditions. However, the fungal communities in the rhizosphere of Xinjiang jujube have never been studied previously.

In this study, we used 454 pyrosequencing to analyze the fungal communities in the jujube rhizosphere in the three areas of Xinjiang, namely, the prefectures of Hetian, Kashi, and Aksu in China. The objectives of this study were to characterize fungal diversity and community composition and to compare fungal communities from different areas and growth years. We also isolated several fungal jujube pathogens. Finally, the relationship between fungal phyla and soil conditions was tested by redundancy analysis (RDA).

\section{Materials and Methods}

2.1. Sample Collection and DNA Extraction. Soil samples were collected from the rhizosphere of jujube (variety Junzao) across the southern Xinjiang Uygur Autonomous Region of China (Figure 1). Eighteen samples were collected from the prefectures of Kashi $(\mathrm{Ka})$, Hetian $(\mathrm{He})$, and Aksu (Ak). Samples were identified as $\mathrm{He}-3, \mathrm{He}-8, \mathrm{Ka}-3, \mathrm{Ka}-8, \mathrm{Ak}-3$, and Ak-8, where the numbers 3 and 8 represent the rhizosphere of 3-and 8-year-old jujube plants. The three areas from which soil samples were collected were separated by distances of about $460-770 \mathrm{~km}$. Jujube trees aged 3 and 8 years were selected at each site, and three soil samples for each age were collected by five-point sampling. All soil samples were placed in separate sterile plastic bags that were immediately placed on ice and then transported to the laboratory where they were stored at $-20^{\circ} \mathrm{C}$ until DNA extraction. The physicochemical parameters of the soil were measured as follows. Soil moisture content was estimated by the oven dry-weight method [23] and pH was determined using a glass electrode meter in a suspension of $1 \mathrm{~g}$ of soil in $5 \mathrm{~mL}$ of distilled water. Available phosphorous $(\mathrm{P})$ was extracted using sodium bicarbonate and then measured by the molybdenum blue method. Available potassium (K) was determined by flame photometry [24]. Available nitrogen $(\mathrm{N})$ was determined by potassium persulfate oxidation [25]. Organic matter content was determined as described by Walkey and Black [26]. Total genomic DNA was extracted from soil samples $(0.4 \mathrm{~g})$ by using the Soil DNA kit (OMEGA, Bio-Tek, USA), according to the manufacturer's instructions. The DNA extracted from three successive rounds of extraction from each sample was pooled prior to PCR.

2.2. PCR Amplification and 454 Pyrosequencing. PCR amplification of the fungal internal transcribed spacer (ITS) rDNA genes from genomic DNA was performed with barcoded primers. The bacterial forward primer was ITS1F (CTTGGTCATTTAGAGGAAGTAA), and the reverse primer was ITS2 (GCTGCGTTCTTCATCGATGC). PCR was performed in a $20 \mu \mathrm{L}$ reaction mixture containing $1 \mu \mathrm{L}$ of DNA template $(10 \mathrm{ng} / \mu \mathrm{L}), 0.4 \mu \mathrm{L}$ of each primer $(10 \mathrm{pmol}), 0.15 \mu \mathrm{L}$ of $\mathrm{Ex}$ Taq (TaKaRa), $2 \mu \mathrm{L}$ of $10 \mathrm{x}$ buffer, $1.6 \mu \mathrm{L}$ of dNTPs $(2.5 \mathrm{mM})$ (TaKaRa), and $13.5 \mu \mathrm{L}$ of $\mathrm{ddH}_{2} \mathrm{O}$. Cycling conditions were as follows: initial denaturation at $98^{\circ} \mathrm{C}$ for $2 \mathrm{~min}$, followed by 35 cycles of $98^{\circ} \mathrm{C}$ for $15 \mathrm{~s}, 56^{\circ} \mathrm{C}$ for $30 \mathrm{~s}$, and $72^{\circ} \mathrm{C}$ for $40 \mathrm{~s}$, with a final extension at $72^{\circ} \mathrm{C}$ for $10 \mathrm{~min}$. PCR products were purified using the TaKaRa Agarose Gel DNA Purification kit and quantified with a NanoDrop. A mixture of purified ITS rDNA amplicons from each soil sample was subjected to pyrosequencing on the 454 GS FLX titanium platform (Roche, Basel, Switzerland) at the National Human Genome Centre of China (Shanghai).

2.3. Processing of Pyrosequencing Data. Pyrosequencing data were processed using Mothur (version 1.25.1) following the Schloss standard operating procedure [27]. Pyrosequencing reads with ambiguous nucleotides, sequences <200 bp in length, one or more primer mismatches, or two or more barcode mismatches were removed and excluded from further analysis. The filtered sequences were then assigned to soil samples according to the corresponding barcodes with a Bespoke Java program. Operational taxonomic units (OTUs) at the $3 \%$ dissimilarity level were determined by comparing the sequences to those in the Silva database (http://www.arbsilva.de/) using Mothur, and the most abundant sequence in each OTU was selected as the representative. Representative sequences were taxonomically classified using a Ribosomal Database Project naive Bayesian rRNA classifier 2.2 [28] with a confidence threshold of 0.8 . The relative proportion of a given phylogenetic group with respect to the entire fungal communitywas defined as the number of sequences affiliated with that group divided by the total number of sequences per sample [29].

2.4. Bioinformatics and Statistical Analysis. An OTU-based analysis was performed to calculate species richness, diversity, and coverage of each rhizosphere sample, with a cutoff of 3\% dissimilarity [29]. The $\mathrm{R}$ software (version 2.14.2; $\mathrm{R}$ Development Core Team 2009) was used to calculate the Shannon-Wiener index, Simpson's Diversity index, and Evenness index of each sample [30]. Rarefaction curves generated by Mothur were used to compare relative levels of bacterial OTU diversity across all soil samples [29]. The coefficient of 


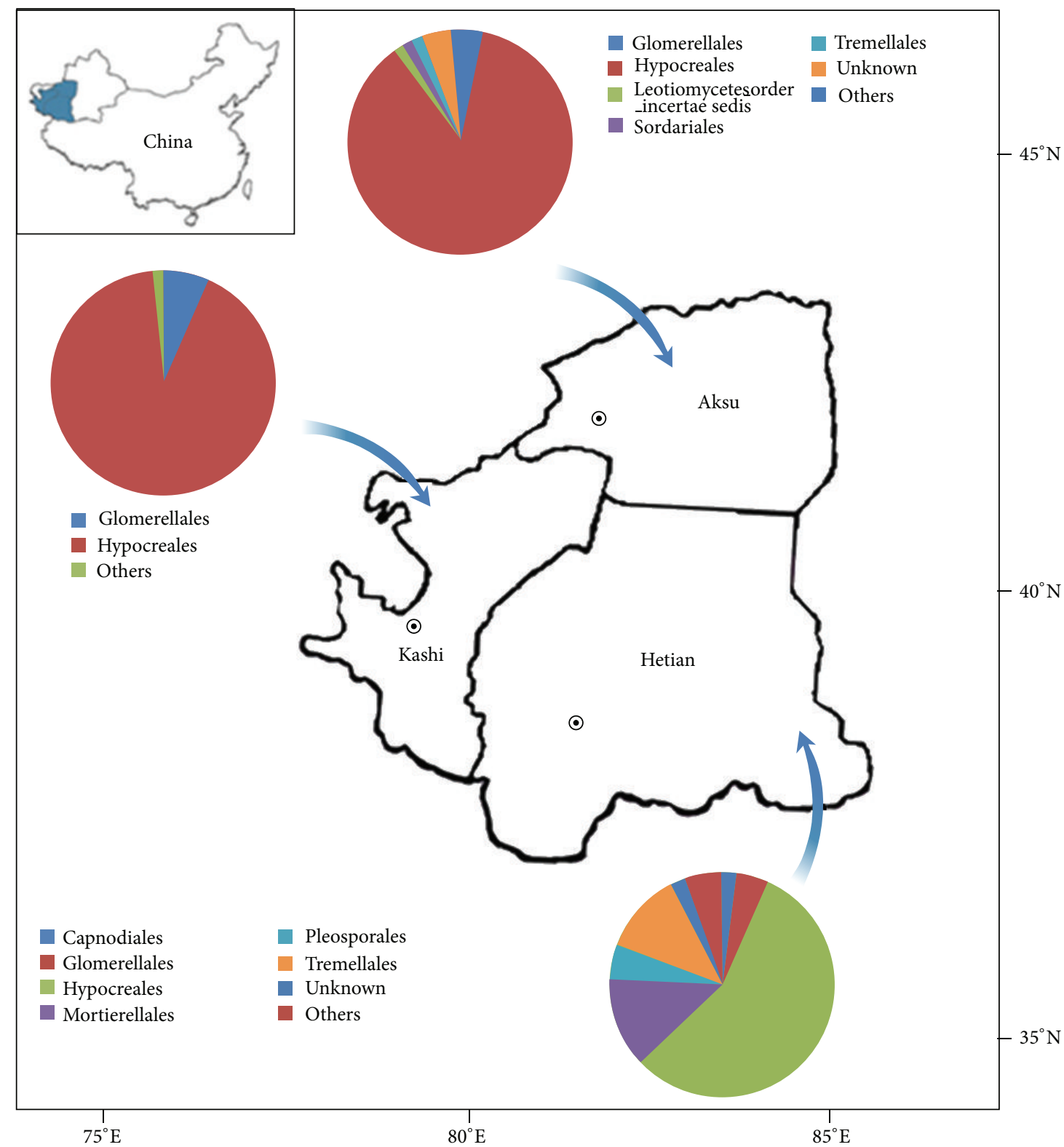

FIGURE 1: Location of sampling sites of soil samples across the Xinjiang Uygur Autonomous Region of China and relative abundance of the dominant fungal order in 6 soil samples.

community structure or distance coefficient was calculated and used for UPGMA cluster analysis. Venn diagrams were generated using custom Perl scripts [31]. The relationship between bacterial phyla and soil conditions was analyzed by redundancy analysis using $\mathrm{R}$ software. All chemical data are expressed as the mean value \pm SE. Analysis of variance was performed using SAS 8.0 to test for sample differences in $\alpha$ diversity and soil properties.

\section{Results}

3.1. Estimators for Diversity and Species Richness of Fungal Communities. For the six sequencing samples, exclusion of low-quality and short sequence reads yielded 40,488 fungal
ITS pyrotag reads. Each sample averaged 6,748 reads; the number of each sample was 6943, 6647, 6584, 6550, 6860, and 6904 . Using a 3\% dissimilarity cut-off for clustering, thereads were grouped into different OTUs (Table 1). The number of fungal OTUs was generally higher in soil samples collected from the rhizosphere of 3-year-old jujube from the same area, with the greatest number of OTUs (474) in sample He-3and the least (189) in sample Ka-8. Rarefaction curves for the fungal community at distance levels of 0.03 had not reached an asymptote (Figure 2). Thus, the sequencing capability did not fully represent the number of different fungal communities, but by combining the rarefaction curves with the Shannon diversity index, we found that, with the increase in sequencing number, the Shannon diversity curves 
TABLE 1: Diversity and richness index of fungal community from 6 soil samples.

\begin{tabular}{llccccccc}
\hline Sites & Location & Sample ID & Cutoff & OTUs & ACE & Chao & Shannon & Coverage \\
\hline \multirow{2}{*}{ Hetian } & $37^{\circ} 07^{\prime} \mathrm{N}$ & He-3 & 0.03 & $474 \pm 14^{\mathrm{a}}$ & $1064.9 \pm 35.2^{\mathrm{a}}$ & $751.1 \pm 35.3^{\mathrm{a}}$ & $4.37 \pm 0.01^{\mathrm{a}}$ & 0.969 \\
& $79^{\circ} 56^{\prime} \mathrm{E}$ & He-8 & 0.03 & $344 \pm 33^{\mathrm{c}}$ & $778.6 \pm 60.5^{\mathrm{c}}$ & $578.0 \pm 46.5^{\mathrm{b}}$ & $3.80 \pm 0.03^{\mathrm{b}}$ & 0.976 \\
\hline \multirow{2}{*}{ Kashi } & $38^{\circ} 11^{\prime} \mathrm{N}$ & Ka-3 & 0.03 & $314 \pm 16^{\mathrm{c}}$ & $788.9 \pm 30.3^{\mathrm{c}}$ & $558.6 \pm 36.1^{\mathrm{b}}$ & $2.98 \pm 0.04^{\mathrm{c}}$ & 0.976 \\
& $77^{\circ} 16^{\prime} \mathrm{E}$ & Ka-8 & 0.03 & $189 \pm 24^{\mathrm{d}}$ & $287.4 \pm 47.1^{\mathrm{d}}$ & $291.7 \pm 68.8^{\mathrm{c}}$ & $2.38 \pm 0.05^{\mathrm{d}}$ & 0.988 \\
\hline \multirow{2}{*}{ Aksu } & $80^{\circ} 11^{\prime} \mathrm{N}$ & Ak-3 & 0.03 & $418 \pm 28^{\mathrm{b}}$ & $1040.9 \pm 51.8^{\mathrm{a}}$ & $763.0 \pm 72.6^{\mathrm{a}}$ & $3.71 \pm 0.02^{\mathrm{b}}$ & 0.970 \\
& $40^{\circ} 47^{\prime} \mathrm{E}$ & Ak-8 & 0.03 & $399 \pm 30^{\mathrm{b}}$ & $927.4 \pm 29.7^{\mathrm{b}}$ & $740.3 \pm 34.9^{\mathrm{a}}$ & $3.60 \pm 0.05^{\mathrm{b}}$ & 0.972 \\
\hline
\end{tabular}

Statistically significant differences $(P<0.05)$ between 6 different soil samples from 3 areas. Different letters $(a, b, c$, and d) in column indicate significant difference $(P<0.05)$ between sampling sites according to Duncan's multiple comparison.

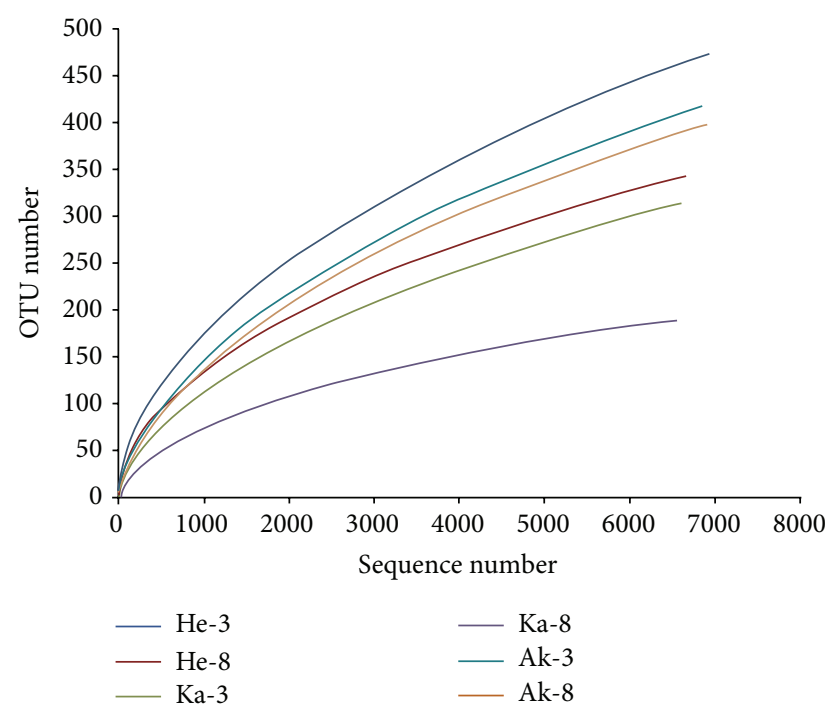

FIGURE 2: Rarefaction curves of fungal depicting the effect of 3\% dissimilarity on the number of OTUs identified in the 6 soil samples.

(Figure 3) approached a plateau. Therefore, the data were sufficient to allow an analysis of fungal communities. The indices of diversity and richness of fungi in soil samples are shown in Table 1. The ACE and Chao values, which are indicators of species richness, were greater for the rhizosphere fungal community of the 3-year-old jujube than those for the 8year-old jujube rhizosphere. Shannon diversity index values showed a similar trend in fungal diversity.

3.2. Fungal Community Composition and Structure Analysis. All fungal sequences were classified at the phylum level down to the genus level according to the Mothur program. Of the classifiable sequences, four phyla were identified (Figure 4), with Ascomycota representing the most dominant phyla and accounting for $73.2 \%, 71.7 \%, 98.9 \%, 97.8 \%, 97.6 \%$, and $97.1 \%$ of the six soil samples. The distribution of Basidiomycota varied, accounting for $20.3 \%$ in sample $\mathrm{He}-8$, but only $0.058 \%$ in Ak-3. Some phyla existed in only specific soil samples. Chytridiomycota was found only in samples $\mathrm{He}-3$ and Ak3. Glomeromycota was present only in $\mathrm{He}-3$ and constituted 1 OTU. In addition, the proportion of unclassified fungi was relatively large in $\mathrm{He}-3$ (21.7\%) and $\mathrm{He}-8$ (8.6\%).

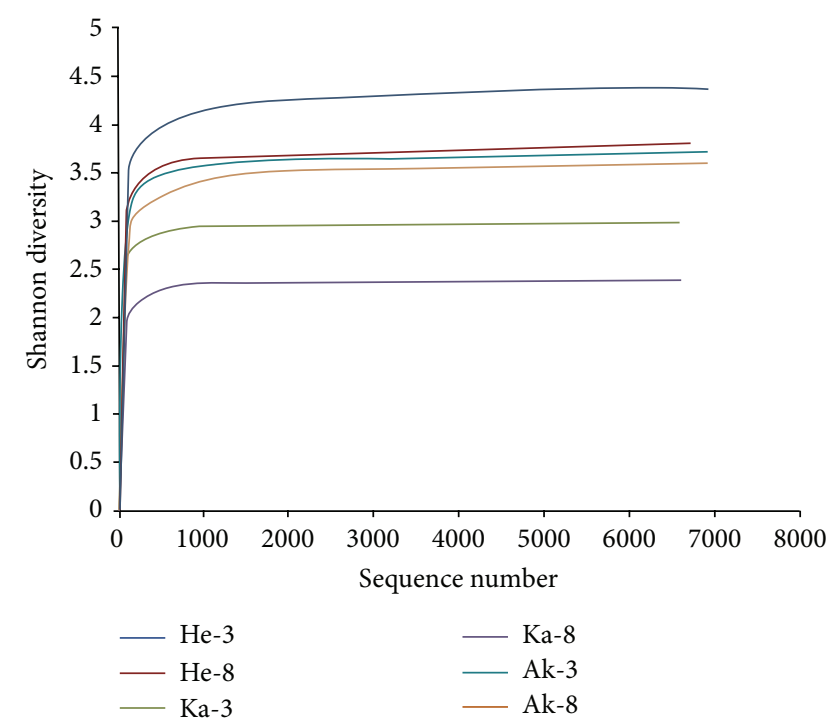

FIGURE 3: Shannon curves of fungal depicting the effect of $3 \%$ dissimilarity on the number of OTUs identified in the 6 soil samples.

Down to the order level, the most abundant fungal order was Hypocreales, accounting for $56.3 \%, 91.9 \%$, and $85.1 \%$ of the three sampling areas. The diversity of fungal order was higher in Hetian than in other areas. The Mortierellales and Tremellales were the next most common orders, at $13.1 \%$ and $11.6 \%$. The Hetian area also included Glomerellales (4.6\%), Pleosporales (4.9\%), Capnodiales $(2.0 \%)$, and unknown (2.2\%). In the Kashi area, Hypocreales accounted for $91.9 \%$, Glomerellales was $6.6 \%$, and others were $1.5 \%$; there were no other fungal orders, making the diversity in this area the lowest of the tested regions. Hypocreales (85.1\%), Tremellales (1.3\%), and Glomerellales (3.2\%) were found in Aksu and Hetian; Leotiomycetes (1.3\%) was found only in Aksu. Mortierellales was absent from Aksu. We also identified jujube pathogens from the fungal sequence reads and found that the fewest pathogens were in the Xinjiang soil samples (Aspergillus and Schizophyllum). Aspergillus was found inHetian and Aksu, only in 3-year-old jujube trees. The proportions of Aspergillus in $\mathrm{He}-3$ and $\mathrm{Ak}-3$ were $0.1 \%$ and $0.4 \%$, respectively. 


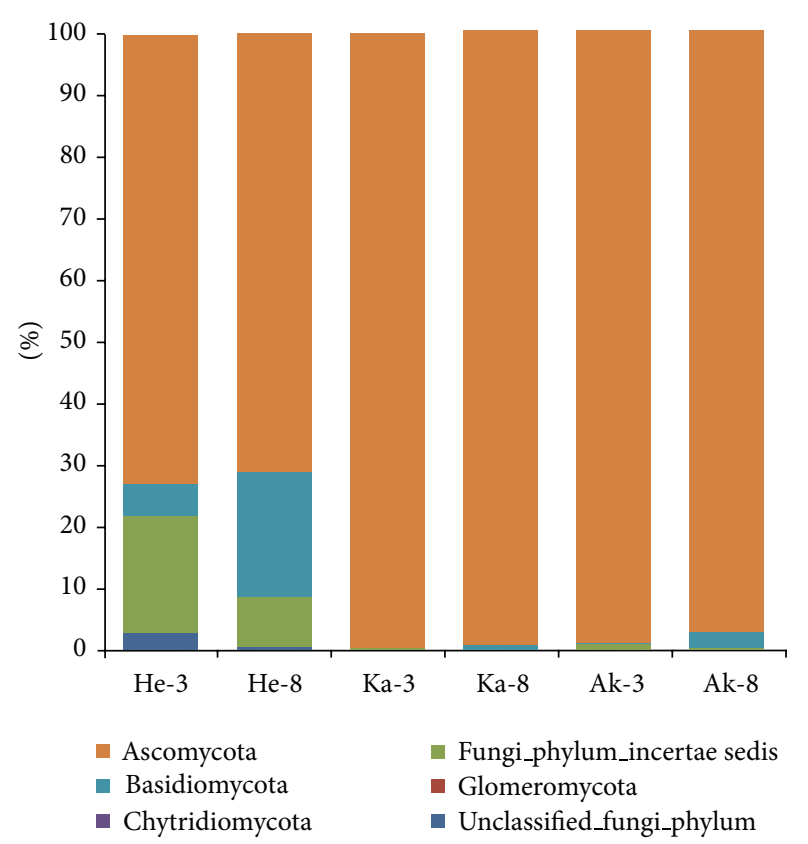

FIgURE 4: Relative abundance of the dominant fungal phyla in 6 soil samples.

\subsection{Comparison of Fungal Communities between Different} Sampling Areas. Fungal OTUs common to the three sampling areas were represented using a Venn diagram to compare the relationships between the three communities (Figure 5). The number of fungal OTUs obtained for each prefecture was as follows: 656 for Hetian, 449 for Kashi, and 653 for Aksu at the level of 3\% dissimilarity. Hetian and Kashi shared 193 OTUs, Hetian and Aksu shared 287, and Kashi and Aksu shared 275. All three sites shared 166 OTUs. Two primary phyla were common to the three areas: Ascomycota and Basidiomycota. Chytridiomycota was present in soil samples from Hetian and Aksu. Glomeromycota was present only in samples from Hetian. Chao and Shannon's indices indicate that the diversity of fungal communities in Hetian was higher than in Kashi and Aksu. We also identified differences between growth ages in the same sampling area. For the soil samples from Hetian prefecture, the dominant phyla were the same for samples He-3 and He-8, except for Chytridiomycota and Glomeromycota, which were identified only in the He-3 sample. However, the dominant phyla had an unbalanced distribution between the 3- and 8-year-old samples. Ascomycota was the most abundant phylum in each sample, accounting for $73.2 \%$ in $\mathrm{Ka}-8$ and $71.1 \%$ in $\mathrm{He}-3$. The proportion of Basidiomycota differed in He-3 (4.32\%) and He-8 (20.3\%).

\subsection{Relationships between Fungal Communities and Environ-} mental Variables. Redundancy analysis revealed a significant correlation between soil properties and the abundance of fungal phyla (Figure 6). The abundance of phyla in He-3 correlated with available $\mathrm{K}$ and $\mathrm{N}$, and the abundance of phyla in He-8 correlated with $\mathrm{pH}$. The fungal communities in other soil samples correlated negatively with organic matter,

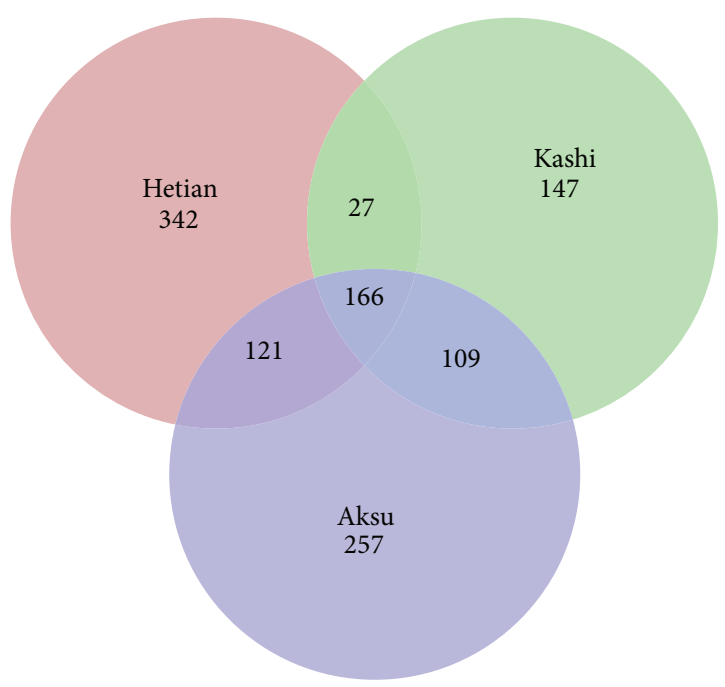

FIGURE 5: Venn diagram representing the number of fungal OTUs that are unique and shared between the samples from 3 different sampling areas.

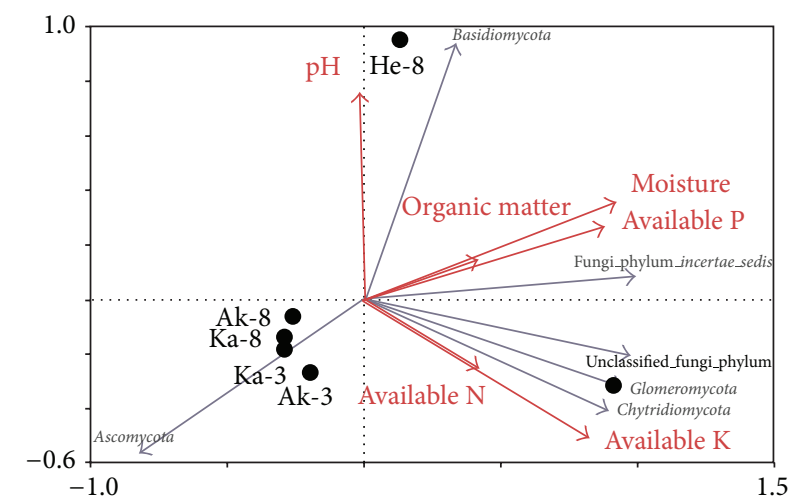

FIGURE 6: Redundancy analysis (RDA) of abundant fungal phyla and soil properties for individual samples from 3 sampling areas.

moisture, and available P. We found that soil organic matter, moisture, and available P had negative effects on Ascomycota abundance and positive effects on the fungi incertae sedis. The abundance of Chytridiomycotaand Glomeromycota correlated with available $\mathrm{K}$ and $\mathrm{N}$ content. Furthermore, the relative abundance of Basidiomycota correlated with soil $\mathrm{pH}$. Redundancy analysis showed that the sampling sites had less influence on the fungal communities than soil properties, supporting environmental variation as the major determinant of fungal community structure.

\section{Discussion}

The rhizosphere is a critical interface that supports the exchange of resources between plants and their associated soil environment [32]. Rhizosphere microorganisms can greatly affect plant growth by transforming nutrients, contributing to soil organic matter formation, and acting as root pathogens or antagonists of root pathogens. In this study, we used the 454 
pyrosequencing technique to investigate rhizospherefungal diversity and community structure at different growth stages of Xinjiang jujube in three major jujube production areas in China.

At present, the internal transcribed spacer (ITS) regions in the $18 \mathrm{~S}, 5.8 \mathrm{~S}, 28 \mathrm{~S}$ ribosomal RNA gene cluster of fungi have been validated as the best DNA barcode marker for fungal species identification and successfully in metagenomic studies [33]. The primer pair (ITS1F, ITS2) in our study is fungal-specific for the ITS1 region and amplifies a fragment of c. $400 \mathrm{bp}$. The sequencing yielded 40,488 ITS rDNA; each sample averaged 6,748 reads, with only 189-474 OTUs identified in each soil sample. The number of reads and OTUs was lower than previous reports; for example, Buée et al. [34] obtained 166,350 reads and 600-1000 OTUs in each soil sample. In this study, none of the rarefaction curves reached saturation, indicating that further sampling would have revealed additional diversity. Our results are in concordance with previous reports $[27,35,36]$; however, the Shannon index curves became saturated, suggesting that increasing the amount of sequencing reads would not help identify fundamentally new species. We thus concluded that our results are an accurate reflection of the fungal communities they represent.

Sequence analyses revealed that the majority of recovered fungal sequences belonged to the Dikary (Ascomycota, Basidiomycota), which were the most abundant phyla in all samples, accounting for $94.5 \%$. Ascomycota was the most abundant phylum (89.7\% of OTUs), whereasBasidiomycota accounted for a much smaller percentage (4.9\%). These results were in agreement with those of Schadt et al. [37] who also found a large proportion of Ascomycota in 125 cloned fungal sequences from soils. In contrast, O'Brien et al. [38] used Sanger sequencing and found the proportion of Basidiomycota to be larger than that of Ascomycota. The Chytridiomycota and Glomeromycota were probably underestimated in our data. Chytridiomycotawas found in samples He-3 (0.1\%) and Ak-3 (0.03\%), while Glomeromycotawas found in $\mathrm{He}-3(0.05 \%)$ and $\mathrm{Ka}-8(0.11 \%)$. In addition, there were large proportions of unclassified fungi in $\mathrm{He}$ 3 and He-8. A collection of curated sequences for fungal identification is urgently needed, or perhaps the database is inadequate. Because there are fewer fungal phyla, we analyzed the fungal community structure down to the order level. Hypocreales was the most abundant fungal order in contrast to Taylor [39], who reported that Capnodiales was the most abundant fungal order and Hypocrealeswas present in low proportion. The large proportion of unknown fungi found in this and other soil surveys reveals the need for a collection of curated sequences for fungal identification. Pathogenic fungi such as Aspergillus and Schizophyllum werealso detected. The highest number of sequences (40 reads) matched Aspergillus, a known pathogen that causes jujube rot. From the RDA, we know that the function of the fungal populations had links with environmental parameters. For example, Aspergillus belongs to Ascomycota which correlated negatively with organic matter, moisture, and available P; therefore we inferred that increasing the soil organic matter, available $\mathrm{P}$, and irrigation can prevent the jujube disease.
Numerous studies have shown that environmental factors shape community structure [40-42]. In our study, an RDA of the environmental variables indicated that $\mathrm{pH}$, available $\mathrm{N}$, available $\mathrm{K}$, available $\mathrm{P}$, organic matter, and moisture were important for the phylogenetic diversity of fungi. A difference in organic matter composition and functioning was reported previously [43]. It is essential to analyze identical numbers of sequences from all samples because diversity estimates always scale up with changes in sampling depth. Our study provides an outline of the fungal diversity and community of the rhizosphere of Xinjiang jujube.

\section{Conclusions}

The aim of this research was to reveal the fungal communities living in the rhizosphere of Xinjiang jujube. 40,488 fungal ITS pyrotag reads were yielded by pyrosequencing. The results revealed the low fungal diversity in this areas. The most abundant fungal phylum was Ascomycota, and the most abundant order was Hypocreales. And the fungal communities were different from other studies. By comparison of fungal communities between different sampling areas, we found that the diversity of fungal communities in Hetian was higher than in Kashi and Aksu. Meanwhile, we discussed the relationship between fungal communities and environmental variables. Furthermore, we hope that our results will provide a reference for future research on the diversity of fungal communities, which thrive in the rhizosphere.

\section{Conflict of Interests}

The authors declare no conflict of interests.

\section{Acknowledgments}

This work was financially supported by The National Special Funds for Nonprofit Research in Forestry Industry (Grant no. 201304212 and Grant no. 201304212). The authors thank Wei Qin, Yin-Peng Guo, and Shou-Quan Yi from Xinjiang Agricultural University for help during sample collection and the measure of soil characterization. The authors also thank Zhi-Wen Wang, Tientsin University, for revising the paper.

\section{References}

[1] T. Větrovský and P. Baldrian, "Analysis of soil fungal communities by amplicon pyrosequencing: current approaches to data analysis and the introduction of the pipeline SEED," Biology and Fertility of Soils, vol. 49, no. 8, pp. 1027-1037, 2013.

[2] M. W. Taylor, P. Tsai, N. Anfang, H. A. Ross, and M. R. Goddard, "Pyrosequencing reveals regional differences in fruit-associated fungal communities," Environmental Microbiology, vol. 16, no. 9, pp. 2848-2858, 2014.

[3] S. J. Morris and G. P. Robertson, "Linking function between scales of resolution," in The Fungal Community, J. Dighton, J. F. White, P. Oudemans, and J. F. White, Eds., pp. 13-26, Marcel Dekker, New York, NY, USA, 2005.

[4] J. L. Green, B. J. M. Bohannan, and R. J. Whitaker, "Microbial biogeography: from taxonomy to traits," Science, vol. 320, no. 5879, pp. 1039-1043, 2008. 
[5] J. Rousk, E. Baath, P. C. Brookes, C. L. Lauber, C. Lozupone, and J. G. Caporas, "Pesticide effects on bacterial diversity in agricultural soils-a review," Microbial Ecology, vol. 33, pp. 443453, 2001.

[6] P. Nannipieri, J. Ascher, M. T. Ceccherini, L. Landi, G. Pietramellara, and G. Renella, "Microbial diversity and soil functions," European Journal of Soil Science, vol. 54, no. 4, pp. 655-670, 2003.

[7] S. M. D. Goldberg, J. Johnson, D. Busam et al., "A sanger/pyrosequencing hybrid approach for the generation of high-quality draft assemblies of marine microbial genomes," Proceedings of the National Academy of Sciences of the United States of America, vol. 103, no. 30, pp. 11240-11245, 2006.

[8] R. Ohtonen, H. Fritze, T. Pennanen, A. Jumpponen, and J. Trappe, "Ecosystem properties and microbial community changes in primary succession on a glacier forefront," Oecologia, vol. 119, no. 2, pp. 239-246, 1999.

[9] N. Fierer, D. Nemergut, R. Knight, and J. M. Craine, "Changes through time: integrating microorganisms into the study of succession," Research in Microbiology, vol. 161, no. 8, pp. 635642, 2010.

[10] E. R. Mardis, "The impact of next-generation sequencing technology on genetics," Trends in Genetics, vol. 24, no. 3, pp. 133-141, 2008.

[11] R. Blaalid, T. Carlsen, S. Kumar et al., "Changes in the rootassociated fungal communities along a primary succession gradient analysed by 454 pyrosequencing," Molecular Ecology, vol. 21, no. 8, pp. 1897-1908, 2012.

[12] A. Jumpponen and K. L. Jones, "Massively parallel 454 sequencing indicates hyperdiverse fungal communities in temperate Quercus macrocarpa phyllosphere," New Phytologist, vol. 184, no. 2, pp. 438-448, 2009.

[13] Y. W. Lim, B. K. Kim, C. Kim et al., "Assessment of soil fungal communities using pyrosequencing," The Journal of Microbiology, vol. 48, no. 3, pp. 284-289, 2010.

[14] M. Buée, M. Reich, C. Murat et al., "454 pyrosequencing analyses of forest soils reveal an unexpectedly high fungal diversity," New Phytologist, vol. 184, no. 2, pp. 449-456, 2009.

[15] A. Jumpponen and K. L. Jones, "Seasonally dynamic fungal communities in the Quercus macrocarpa phyllosphere differ between urban and nonurban environments," New Phytologist, vol. 186 , no. 2 , pp. 496-513, 2010.

[16] M. Margulies, M. Egholm, W. E. Altman et al., "Genome sequencing in microfabricated high-density picolitre reactors," Nature, vol. 437, pp. 376-380, 2005.

[17] Y. W. Lim, B. K. Kim, C. Kim et al., "Assessment of soil fungal communities using pyrosequencing," Journal of Microbiology, vol. 48, no. 3, pp. 284-289, 2010.

[18] L. Tedersoo, R. H. Nilsson, K. Abarenkov et al., “454 pyrosequencing and Sanger sequencing of tropical mycorrhizal fungi provide similar results but reveal substantial methodological biases," New Phytologist, vol. 188, no. 1, pp. 291-301, 2010.

[19] J. Wu, W. Liu, H. Zeng, L. Ma, and R. Bai, "Water quantity and quality of six lakes in the Arid Xingjiang Region, NW China," Environmental Processes, vol. 1, no. 2, pp. 115-125, 2014.

[20] W. Zhang, X. Long, X. Huo, Y. Chen, and K. Lou, "16S rRNAbased PCR-DGGE analysis of actinomycete communities in fields with continuous cotton cropping in Xinjiang, China," Microbial Ecology, vol. 66, no. 2, pp. 385-393, 2013.

[21] L. Feng-gang, L. Wang, L. Yan-nan, L. Yang-yang, Z. Haiyan, and Z. Li-li, "Analysis of culturable fungal diversity in ehizosphere soil of healthy and diseased cotton in Southern Xinjiang," African Journal of Microbiology Research, vol. 6, pp. 7357-7364, 2012.

[22] J.-W. Li, L.-P. Fan, S.-D. Ding, and X.-L. Ding, "Nutritional composition of five cultivars of Chinese jujube," Food Chemistry, vol. 103, no. 2, pp. 454-460, 2007.

[23] C. D. Moodie, H. W. Smith, and R. L. Hausanbuiler, Laboratory Manual for Soil Fertility, Washington State University Department of Agronomy Pullman, Washington, DC, USA, 1963.

[24] J. Zhao, R. Zhang, C. Xue et al., "Pyrosequencing reveals contrasting soil bacterial diversity and community structure of two main winter wheat cropping systems in China," Microbial Ecology, vol. 67, no. 2, pp. 443-453, 2014.

[25] M. L. Jackson, Soil Chemical Analysis, Prentice Hall, Englewood Cliffs, NJ, USA, 1962.

[26] A. Walkey and I. A. Black, "An examination of the Degtjareff method for determining soil organic matter and a proposed modification of the chromic acid titration method," Soil Science, vol. 37, no. 1, pp. 29-38, 1934.

[27] O. O. Lee, Y. Wang, J. Yang, F. F. Lafi, A. Al-Suwailem, and P.Y. Qian, "Pyrosequencing reveals highly diverse and speciesspecific microbial communities in sponges from the Red Sea," The ISME Journal, vol. 5, no. 4, pp. 650-664, 2011.

[28] W. Jie, L. Lite, and D. Yang, "The correlation between freezing point and soluble solids of fruits," Journal of Food Engineering, vol. 60 , no. 4, pp. 481-484, 2003.

[29] J. Zhao, R. Zhang, C. Xue et al., "Pyrosequencing reveals contrasting soil bacterial diversity and community structure of two main winter wheat cropping systems in China," Microbial Ecology, vol. 67, no. 2, pp. 443-453, 2014.

[30] M. Hu, X. Wang, X. Wen, and Y. Xia, "Microbial community structures in different wastewater treatment plants as revealed by 454-pyrosequencing analysis," Bioresource Technology, vol. 117, pp. 72-79, 2012.

[31] Y.-X. Zeng, M. Yan, Y. Yu et al., "Diversity of bacteria in surface ice of Austre Lovénbreen glacier, Svalbard," Archives of Microbiology, vol. 195, no. 5, pp. 313-322, 2013.

[32] J. A. Peiffer, A. Spor, O. Koren et al., "Diversity and heritability of the maize rhizosphere microbiome under field conditions," Proceedings of the National Academy of Sciences of the United States of America, vol. 110, no. 16, pp. 6548-6553, 2013.

[33] K. A. Seifert, "Integrating DNA barcoding into the mycological sciences," Persoonia, vol. 21, pp. 162-166, 2008.

[34] M. Buée, M. Reich, C. Murat et al., “454 pyrosequencing analyses of forest soils reveal an unexpectedly high fungal diversity," New Phytologist, vol. 184, no. 2, pp. 449-456, 2009.

[35] L. F. W. Roesch, R. R. Fulthorpe, A. Riva et al., "Pyrosequencing enumerates and contrasts soil microbial diversity," The ISME Journal, vol. 1, no. 4, pp. 283-290, 2007.

[36] L. N. Lemos, R. R. Fulthorpe, E. W. Triplett, and L. F. W. Roesch, "Rethinking microbial diversity analysis in the high throughput sequencing era," Journal of Microbiological Methods, vol. 86, no. 1, pp. 42-51, 2011.

[37] C. W. Schadt, A. P. Martin, D. A. Lipson, and S. K. Schmidt, "Seasonal dynamics of previously unknown fungal lineages in tundra soils," Science, vol. 301, no. 5638, pp. 1359-1361, 2003.

[38] H. E. O’Brien, J. L. Parrent, J. A. Jackson, J.-M. Moncalvo, and R. Vilgalys, "Fungal community analysis by large-scale sequencing of environmental samples," Applied and Environmental Microbiology, vol. 71, no. 9, pp. 5544-5550, 2005. 
[39] A. F. S. Taylor, "Fungal diversity in ectomycorrhizal communities: sampling effort and species detection," Plant and Soil, vol. 244, no. 1-2, pp. 19-28, 2002.

[40] J. Rousk, E. Bååth, P. C. Brookes et al., "Soil bacterial and fungal communities across a $\mathrm{pH}$ gradient in an arable soil," ISME Journal, vol. 4, no. 10, pp. 1340-1351, 2010.

[41] B. F. T. Brockett, C. E. Prescott, and S. J. Grayston, "Soil moisture is the major factor influencing microbial community structure and enzyme activities across seven biogeoclimatic zones in Western Canada," Soil Biology and Biochemistry, vol. 44, no. 1, pp. 9-20, 2012.

[42] C. L. Lauber, M. S. Strickland, M. A. Bradford, and N. Fierer, "The influence of soil properties on the structure of bacterial and fungal communities across land-use types," Soil Biology \& Biochemistry, vol. 40, no. 9, pp. 2407-2415, 2008.

[43] J. Moukoumi, C. Munier-Lamy, J. Berthelin, and J. Ranger, "Effect of tree species substitution on organic matter biodegradability and mineral nutrient availability in a temperate topsoil," Annals of Forest Science, vol. 63, no. 7, pp. 763-771, 2006. 

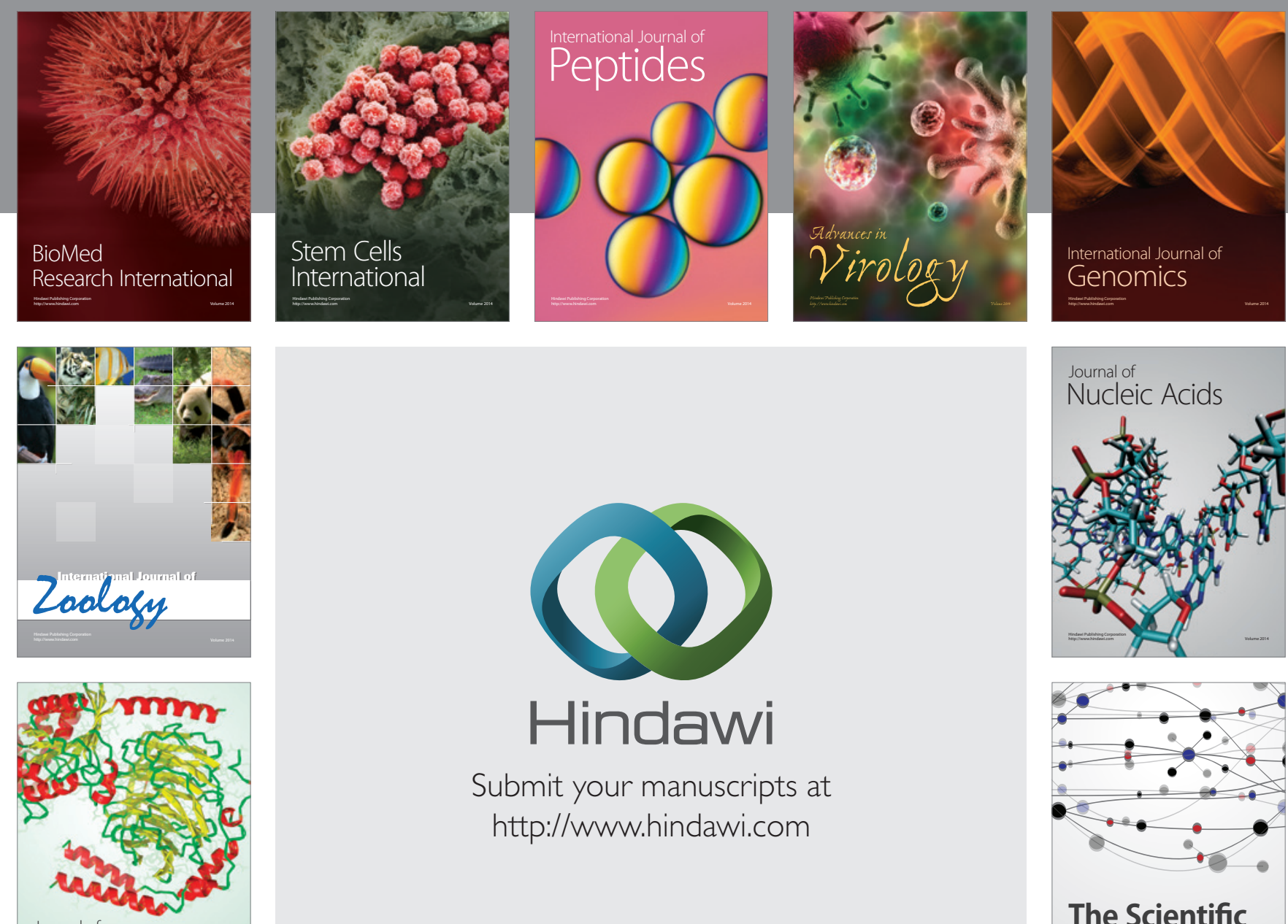

Submit your manuscripts at

http://www.hindawi.com

Journal of
Signal Transduction
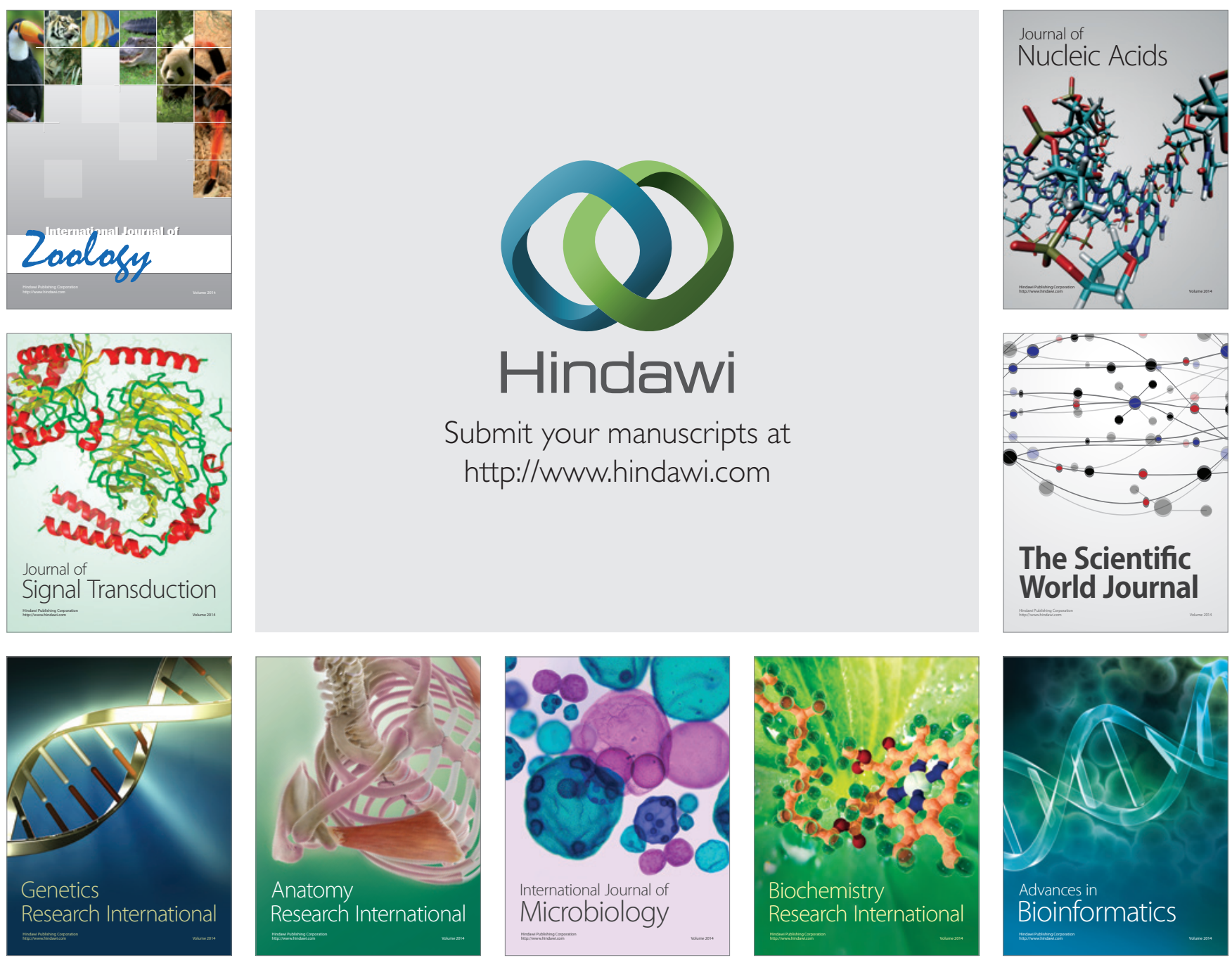

The Scientific World Journal
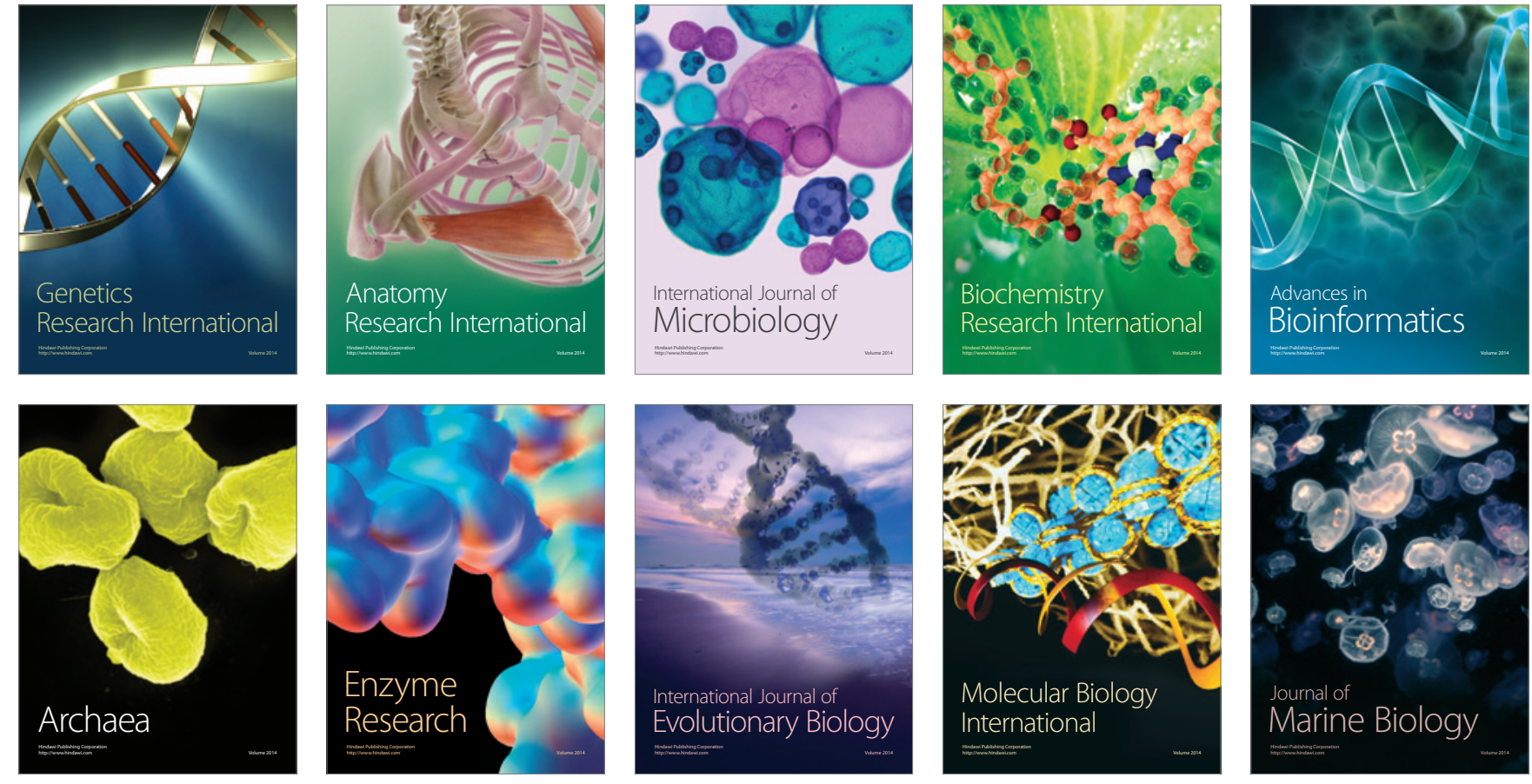